\title{
Absorption-reduced waveguide structure for efficient terahertz generation
}

\author{
L. Pálfalvi, ${ }^{1, a)}$ J. A. Fülöp, ${ }^{2,3}$ and J. Hebling ${ }^{1,2,3}$ \\ ${ }^{1}$ Institute of Physics, University of Pécs, Ifjúság ú. 6, 7624 Pécs, Hungary \\ ${ }^{2}$ MTA-PTE High-Field Terahertz Research Group, Ifjúság ú. 6, 7624 Pécs, Hungary \\ ${ }^{3}$ Szentágothai Research Centre, University of Pécs, Ifjúság ú. 20, 7624 Pécs, Hungary
}

(Received 15 August 2015; accepted 20 November 2015; published online 9 December 2015)

\begin{abstract}
An absorption-reduced planar waveguide structure is proposed for increasing the efficiency of terahertz $(\mathrm{THz})$ pulse generation by optical rectification of femtosecond laser pulses with tiltedpulse-front in highly nonlinear materials with large absorption coefficient. The structure functions as waveguide both for the optical pump and the generated $\mathrm{THz}$ radiation. Most of the $\mathrm{THz}$ power propagates inside the cladding with low $\mathrm{THz}$ absorption, thereby reducing losses and leading to the enhancement of the $\mathrm{THz}$ generation efficiency by up to more than one order of magnitude, as compared with a bulk medium. Such a source can be suitable for highly efficient THz pulse generation pumped by low-energy $(\mathrm{nJ}-\mu \mathrm{J})$ pulses at high $(\mathrm{MHz})$ repetition rates delivered by compact fiber lasers. C 2015 AIP Publishing LLC. [http://dx.doi.org/10.1063/1.4937347]
\end{abstract}

The recent years witnessed a rapid development of pulsed terahertz $(\mathrm{THz})$ sources driven by femtosecond lasers, which became an enabling tool for new research directions such as nonlinear $\mathrm{THz}$ spectroscopy. THz sources with high peak intensity typically require pump pulses with mJ-level energy, thereby usually limiting the pulse repetition rate to the $\mathrm{kHz}$ range. Linear and nonlinear $\mathrm{THz}$ spectroscopic studies and other applications highly benefit from increased repetition rate, and there is a strong need to develop efficient $\mathrm{THz}$ sources with significantly higher $(1-100 \mathrm{MHz})$ repetition rates. Fiber laser technology provides commercially available sources with superior stability and compactness delivering femtosecond pulses at high repetition rates but with moderate pulse energy (up to the $10-\mu \mathrm{J}$ level). It is challenging to design efficient $\mathrm{THz}$ sources based on optical rectification (OR) at these small or moderate pump pulse energies.

The efficiency of OR can be increased by cylindrical focusing, or by using waveguide or waveguide-like structures. $^{1-9}$ In case of materials with high nonlinearity but strong absorption in the $\mathrm{THz}$ range, such as $\mathrm{LiNbO}_{3}$ (LN) with $d_{33}=168 \mathrm{pm} / \mathrm{V}$ nonlinear optical coefficient ${ }^{10}$ and tens of $\mathrm{cm}^{-1}$ absorption coefficient, ${ }^{11}$ it is important to suppress absorption. A promising way to achieve this is to sandwich a few $\mu \mathrm{m}$ thick LN layer between layers of significantly smaller absorption. ${ }^{1-5}$ A suitable material for such a lowabsorption cladding is silicon ( $\mathrm{Si}$ ). The structures reported so far are guiding only the optical pump; ${ }^{1-5}$ the selected material parameters and dimensions do not allow wave guiding in the $\mathrm{THz}$ range. Consequently, the $\mathrm{THz}$ radiation leaves the LN layer through its boundary to the cladding. This reduces the propagation length of the generated $\mathrm{THz}$ radiation inside LN, thereby minimizing absorption losses. However, because of the reduced interaction length, the efficiency of $\mathrm{THz}$ generation is lower than it could be for a (velocitymatched) structure guiding both pump and THz. In the former case it is the intensity, while in the latter case it is the

\footnotetext{
${ }^{\text {a) }}$ Author to whom correspondence should be addressed. Electronic mail: palfalvi@fizika.ttk.pte.hu
}

field strength of the $\mathrm{THz}$ components generated at different locations, which is summed up. Latter can enable to achieve a significantly higher $\mathrm{THz}$ generation efficiency. Although such double-waveguide structures were already introduced for phase matching of $\mathrm{THz}$ generation by parametric interaction $^{6}$ or optical rectification, ${ }^{8}$ the problem of absorption was nowhere mentioned in these works.

In this work, an absorption-reduced waveguide (ARWG) $\mathrm{THz}$ source is introduced where both the optical pump as well as the generated $\mathrm{THz}$ radiation are guided such that velocity matching is fulfilled for OR. The key point for efficient $\mathrm{THz}$ generation is that the $\mathrm{THz}$ waveguide has to have a cladding with orders of magnitude smaller absorption coefficient in the $\mathrm{THz}$ range than that of the core. Appropriate waveguide design ensures that $80 \%-95 \%$ of the $\mathrm{THz}$ energy propagates in the cladding. The absorption losses decrease with increasing fraction of power propagating in the cladding. Velocity matching is achieved by using the tilted-pulse-front pumping (TPFP) technique. ${ }^{12}$

The proposed absorption-reduced planar waveguide $\mathrm{THz}$ source consists of a common core for both the optical pump and the $\mathrm{THz}$ radiation (Fig. 1(a)), forming also the nonlinear medium for OR. We assume $0.67 \mathrm{~mol}$. \% MgOdoped stoichiometric $\mathrm{LN}$ as the core material, but other materials are also possible (e.g., ZnTe, GaP, CdTe, and DAST), where the absorption may originate from the usual
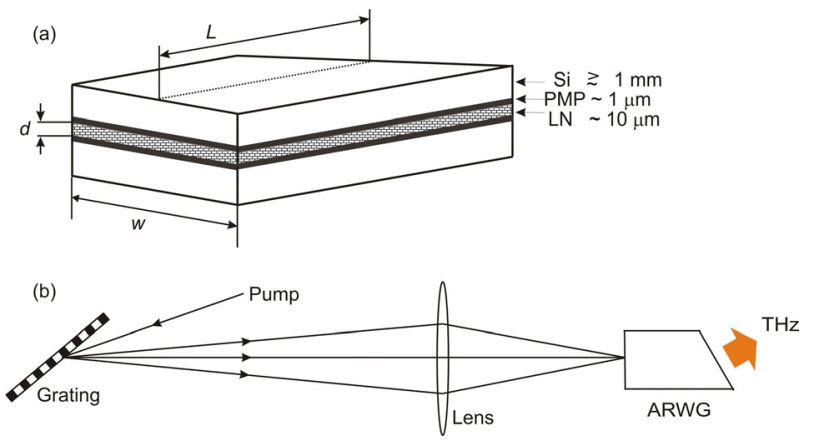

FIG. 1. (a) The ARWG structure. (b) The ARWG THz source with TPFP (top view). 
TABLE I. Optical refractive $\left(n_{\mathrm{p}}\right)$, optical group $\left(n_{\mathrm{g}, \mathrm{p}}\right)$, and THz refractive $\left(n_{\mathrm{THz}}\right)$ indices, and the $\mathrm{THz}$ absorption coefficients $\left(\alpha_{\mathrm{THz}}\right)$ for the materials used in the ARWG.

\begin{tabular}{lcccc}
\hline \hline & $n_{\mathrm{p}}(1 \mu \mathrm{m})$ & $n_{\mathrm{g}, \mathrm{p}}(1 \mu \mathrm{m})$ & $n_{\mathrm{THz}}(1 \mathrm{THz})$ & $\alpha_{\mathrm{THz}}(1 \mathrm{THz})$ \\
\hline LN & 2.15 (Ref. 14) & 2.21 (Ref. 14) & 4.96 (Ref. 11) & $27.3 \mathrm{~cm}^{-1}$ (Ref. 11) \\
PMP & 1.47 (Ref. 15) & not relevant & 1.46 (Ref. 15) & $\sim 1.1 \mathrm{~cm}^{-1}$ (Ref. 15) \\
$\mathrm{Si}$ & $\sim 3.5$ (Ref. 5) & not relevant & 3.42 (Ref. 16) & $\sim 0.05 \mathrm{~cm}^{-1}$ (Ref. 16) \\
\hline \hline
\end{tabular}

complex dielectric function or from free-carrier absorption generated by multiphoton absorption of the pump. LN has extremely large nonlinearity, but also significant absorption in the $\mathrm{THz}$ range (Table I), which reduces the pump-to- $\mathrm{THz}$ conversion efficiency. ${ }^{13}$ A suitable cladding material for guiding $\mathrm{THz}$ radiation generated in the $\mathrm{LN}$ core is silicon (Si). The absorption coefficient of $\mathrm{Si}$ is more than two orders of magnitude smaller than that of LN (Table I). The THz refractive indices of both $\mathrm{LN}$ and $\mathrm{Si}$ are larger than the group index of LN at the typical pump wavelength of $1 \mu \mathrm{m}$ (Table I). In this situation, velocity matching can be achieved by TPFP in the plane of the LN core (Fig. 1(b)). Owing to the mode structure of the THz field in the Si-LN$\mathrm{Si}$ waveguide (see also Fig. 3 inset), in practical situations (see below) it is enough if the thickness of the Si cladding is about $1 \mathrm{~mm}$ or larger.

As mentioned earlier, the ARWG THz source has to guide both the optical pump and the THz waves. However, for typical pump wavelengths, the refractive index of the Si cladding is larger than that of the LN core (Table I), which prohibits wave guiding in the optical range. To solve this problem (similarly to Ref. 5) an inner cladding was introduced between the LN core and the Si outer cladding (Fig. 1(a)). Suitable materials for the inner cladding are, for example, polymers having smaller optical refractive index than that of the LN core and low THz absorption. ${ }^{15,17}$ It should also be transparent in the optical wavelength range of interest. One suitable material is polymethylpentene (PMP, often referred to as TPX), frequently used for $\mathrm{THz}$ applications (Table I). The thickness of the PMP inner cladding is advantageously chosen in the same order of magnitude as the pump wavelength. By this choice the influence of the PMP layer on the THz wave is negligible. At the same time it also prohibits the penetration of the optical pump into the Si outer cladding, since its penetration depth into the PMP is by one order of magnitude smaller than the PMP thickness. This is important in order to avoid free-carrier generation in $\mathrm{Si}$, which could induce absorption in the $\mathrm{THz}$ range. Besides creating the necessary pulse front tilt for velocity matching, the TPFP setup has to provide mode matching of the incoming pump beam to the lowest-order mode of the inner PMP-LN-PMP waveguide. This can be easily achieved by inserting suitable (cylindrical) lenses either in front of or behind the grating.

Numerical simulations were carried out to explore the performance of the ARWG THz source. In the calculations, the influence of the PMP inner cladding on the $\mathrm{THz}$ wave was neglected due to its small absorption coefficient and small thickness. As mentioned earlier, the thickness of Si layer should be at least $1 \mathrm{~mm}$ in practice, which allows to neglect $\mathrm{THz}$ waveguide effects of the Si-air outer boundary. Hence, for the sake of simplicity, in the simulations a Si-LN-Si waveguide with an LN core of thickness $d$ and a Si cladding of infinitely large thickness was considered. Waveguide dispersion for the pump was neglected. A pump wavelength of $1030 \mathrm{~nm}$ was assumed, which is typical for $\mathrm{Yb}$-doped (fiber) lasers.

By applying Eqs. (7.2)-(20) of Ref. 18 for the lowestorder TE mode, one can obtain for the core thickness the following expression:

$$
d=\frac{c}{\pi \nu n_{\mathrm{eff}, \mathrm{THz}} \sqrt{\left(\frac{n_{1}}{n_{\mathrm{eff}, \mathrm{THz}}}\right)^{2}-1}} \tan ^{-1} \sqrt{\frac{1-\left(\frac{n_{2}}{n_{\mathrm{eff}, \mathrm{THz}}}\right)^{2}}{\left(\frac{n_{1}}{n_{\mathrm{eff}, \mathrm{THz}}}\right)^{2}-1}} .
$$

Here, $c$ is the speed of light in vacuum, $n_{1}$ and $n_{2}$ are the refractive indices at the $\mathrm{THz}$ frequency $\nu$ of core and cladding, respectively, and $n_{\mathrm{eff}, \mathrm{THz}}$ is the desired effective refractive index with $n_{\mathrm{Si}, \mathrm{THz}}<n_{\mathrm{eff}, \mathrm{THz}}<n_{\mathrm{LN}, \mathrm{THz}}$. The necessary core thickness was plotted versus $n_{\text {eff,THz }}$ at a few selected frequencies between $0.5 \mathrm{THz}$ and $2.5 \mathrm{THz}$ in Fig. 2(a), and versus the $\mathrm{THz}$ frequency keeping the effective $\mathrm{THz}$ index of refraction at the constant value of $n_{\text {eff }, \mathrm{THz}}=3.8$ in Fig. 2(b).

Fig. 3 shows the power confinement ratio $P_{\text {core }} / P_{\text {total }}$ of the $\mathrm{THz}$ power accommodated inside the $\mathrm{LN}$ core and the total $\mathrm{THz}$ power, calculated from the transversal intensity distribution (see Chap. 7.2 of Ref. 18) of the lowest-order waveguide mode as a function of $n_{\mathrm{eff}, \mathrm{THz}} . P_{\text {core }} / P_{\text {total }}$ varies between $2 \%$ and $26 \%$ in the $n_{\text {eff }, \mathrm{THz}}=3.5$ to 4.0 range, independently from the $\mathrm{THz}$ frequency. These power ratio values are sufficiently low to support efficient $\mathrm{THz}$ generation even in highly absorbing core materials such as LN. It is also obvious from Fig. 3 that smaller $n_{\text {eff, } \mathrm{THz}}$ values are more advantageous for reducing the effect of $\mathrm{THz}$ absorption.

The pulse front tilt angle $\gamma$ needed for velocity matching of pump and $\mathrm{THz}$ can be determined from the equation
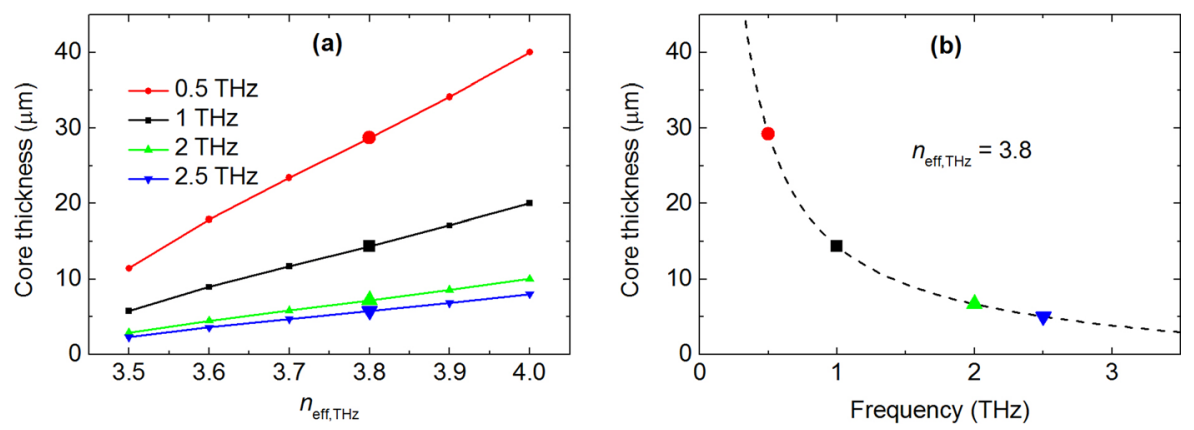

FIG. 2. Required core thickness of the $\mathrm{Si}-\mathrm{LN}-\mathrm{Si} \mathrm{THz}$ waveguide versus the aimed effective $\mathrm{THz}$ refractive index for different $\mathrm{THz}$ frequencies (a) and versus the $\mathrm{THz}$ frequency for $n_{\mathrm{eff}, \mathrm{THz}}$ $=3.8$ (b). The large symbols of Figure 2(a) correspond to those in Figure 2(b). 

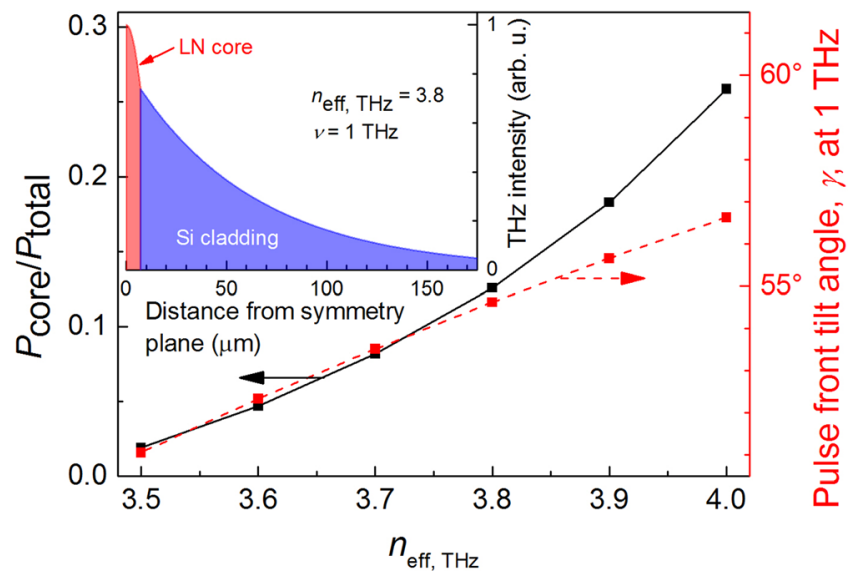

FIG. 3. The power confinement ratio $P_{\text {core }} / P_{\text {total }}$ (black solid line) and the pulse front tilt angle for $1 \mathrm{THz}$ (red dashed line) versus the effective $\mathrm{THz}$ refractive index. The inset shows the $\mathrm{THz}$ intensity distribution for $n_{\mathrm{eff}, \mathrm{THz}}=3.8$ versus the distance measured from the symmetry plane of the structure for $1 \mathrm{THz}$.

$c / n_{\mathrm{eff}, \mathrm{THz}}=\left(c / n_{\mathrm{g}, \mathrm{pump}}\right) \cos \gamma^{12}$ The dependence of $\gamma$ on $n_{\text {eff,THz }}$, assuming $1 \mathrm{THz}$ frequency, is also shown in Fig. 3. Its value varies between $51^{\circ}$ and $57^{\circ}$ and is smaller than $\sim 63^{\circ}$, the value in bulk LN. This is advantageous for increasing the efficiency of the $\mathrm{THz}$ source, since a smaller pulse front tilt angle results in larger pump dispersion length and, consequently, in larger effective interaction length for $\mathrm{THz}$ generation. ${ }^{13}$ Besides the reduced absorption, this also contributes to the larger $\mathrm{THz}$ generation efficiency, as illustrated in Fig. 4 for OR of 10-nJ, 100-fs pulses (more details of the calculation are given later; see also Table II). Fig. 4(a) shows the variation of the pump pulse duration and Fig. 4(b) the build-up of the THz field, in terms of efficiency, along the propagation distance. The maximum efficiency is almost 20 times larger for the ARWG than for bulk LN. The effective interaction length can be defined as the distance needed to reach the maximum efficiency from $5 \% .^{13}$ A smaller $n_{\text {eff,THz }}$ value is more advantageous also in this respect, as it gives a smaller $\gamma$.

Table II summarizes possible ARWG design parameters for various typical laser parameters, together with the basic characteristics of the predicted $\mathrm{THz}$ output. To estimate the THz output, a similar model was used as in Ref. 19. The variation of the pump pulse duration during propagation owing to

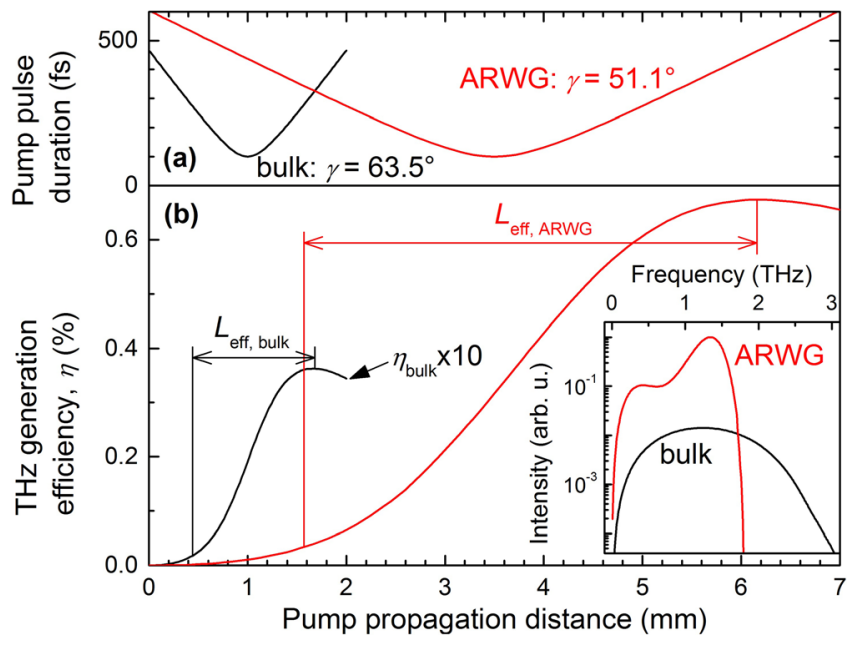

FIG. 4. Pump pulse duration (a) and the (cumulative) THz generation efficiency (b) vs. pump propagation distance for bulk and ARWG. The inset shows the $\mathrm{THz}$ spectra corresponding to the efficiency peaks.

angular and material dispersion in LN was taken into account. A frequency-dependent effective refractive index was considered, and an effective absorption coefficient in the THz range was defined as $\alpha_{\mathrm{eff}, \mathrm{THz}}=\alpha_{\mathrm{THz}} \cdot P_{\text {core }} / P_{\text {total }}$, where $\alpha_{\mathrm{THz}}$ is the absorption coefficient of bulk LN. Waveguide dispersion at the pump wavelength $(1030 \mathrm{~nm})$ was neglected for simplicity. This is justified by the practical limit of about $5 \mathrm{~mm}$ to the ARWG length, which is due to the small core thickness. For a $5 \mathrm{~mm}$ long ARWG with the worst case of $5 \mu \mathrm{m}$ thickness and only $100 \mathrm{fs}$ pulse duration, waveguide dispersion lengthens the pump pulse duration only by 6 fs. The ARWG length was approximately set to the effective interaction length for $\mathrm{THz}$ generation, ${ }^{13,19}$ or to the $5 \mathrm{~mm}$ maximum, to maximize the output $\mathrm{THz}$ energy. In each case, the LN core thickness was chosen to fit with the $\mathrm{THz}$ frequency of the spectral peak that of the bulk source, in order to facilitate a fair comparison. The core width was set to a minimum such that the peak pump intensity remained below $100 \mathrm{GW} / \mathrm{cm}^{2}$ or the average below $\sim 40 \mathrm{~kW} / \mathrm{cm}^{2}$ (measured damage threshold at $300 \mathrm{kHz}$ repetition rate $^{20}$ ), whichever was smaller.

The calculations predict an enhancement of the $\mathrm{THz}$ generation efficiency, $\eta_{\mathrm{ARWG}} / \eta_{\text {bulk }}$, by more than a factor of 20 for $10 \mathrm{~nJ}$ pump pulse energy, as compared with bulk LN. At higher pump energies the enhancement factor is gradually

TABLE II. Design parameters and predicted performance of the ARWG THz source for various laser types.

\begin{tabular}{|c|c|c|c|c|c|}
\hline \multirow[t]{6}{*}{ Pump } & Energy $(\mu \mathrm{J})$ & 0.01 & 0.1 & 1 & 5 \\
\hline & Repetition rate (MHz) & 100 & 10 & 1 & 1 \\
\hline & Pulse duration (fs) & 100 & 200 & 300 & 300 \\
\hline & Average power (W) & 1 & 1 & 1 & 5 \\
\hline & Average intensity $\left(\mathrm{kW} / \mathrm{cm}^{2}\right)$ & 40 & 40 & 30 & 30 \\
\hline & Peak intensity $\left(\mathrm{GW} / \mathrm{cm}^{2}\right)$ & 4 & 20 & 100 & 100 \\
\hline \multirow[t]{3}{*}{ ARWG } & LN core thickness $(\mu \mathrm{m})$ & 5 & 9 & 15 & 15 \\
\hline & Minimum LN core width $(w)(\mathrm{mm})$ & 1.8 & 1.0 & 0.8 & 4.1 \\
\hline & ARWG length $(L)(\mathrm{mm})$ & 5 & 5 & 5 & 5 \\
\hline \multirow[t]{5}{*}{$\mathrm{THz}$} & THz spectral peak (THz) & 1.35 & 0.92 & 0.71 & 0.71 \\
\hline & THz energy (nJ) & $6.6 \times 10^{-2}$ & 3.2 & 95 & 477 \\
\hline & THz average power (mW) & 6.6 & 32 & 95 & 477 \\
\hline & Efficiency enhancement factor $\left(\eta_{\mathrm{ARWG}} / \eta_{\text {bulk }}\right)$ & $22 \times$ & $6.6 \times$ & $2.5 \times$ & $2.5 \times$ \\
\hline & Spectral intensity enhancement factor & $53 \times$ & $18 \times$ & $7.6 \times$ & $7.6 \times$ \\
\hline
\end{tabular}


reduced. It is about 2.5 for $\mu \mathrm{J}$-level energies. Clearly, this reduction is due to the limited ARWG length, since the assumed $300 \mathrm{fs}$ pulse duration would allow effective interaction lengths significantly longer than $5 \mathrm{~mm}$. It is possible to accommodate even larger pump energies over the crosssection of the ARWG, but this may require to increase the core thickness, leading to larger $n_{\text {eff, THz }}$, smaller $P_{\text {core }} / P_{\text {total }}$, and lower $\mathrm{THz}$ generation efficiency. Here, a more in-depth analysis would require to consider wave guiding and possible multi-mode propagation of the pump, but this is out of the scope of the present discussion. We note that even in case of the largest predicted efficiencies $(\sim 10 \%)$, the simple model of OR used here can still be adequate, rather than a more complex one taking into account the influence of the $\mathrm{THz}$ field on the pump, ${ }^{21}$ as the THz field strength is significantly reduced by the penetration into the cladding.

A comparison of the peak $\mathrm{THz}$ spectral intensities of the ARWG and bulk cases reveals an enhancement of $53 \times$ for $10 \mathrm{~nJ}$ pump and $7.6 \times$ at the $\mu \mathrm{J}$-level (Table II), much larger than the efficiency enhancement. The reason for this is the larger dispersion of $n_{\mathrm{eff}, \mathrm{THz}}$ than that of the bulk refractive index of $\mathrm{LN}$, which leads to a reduced $\mathrm{THz}$ spectral width for the ARWG, clearly visible in the inset of Fig. 4.

In summary, an absorption-reduced planar waveguide structure driven by TPFP was proposed for increasing the efficiency of $\mathrm{THz}$ pulse generation by $\mathrm{OR}$ of femtosecond laser pulses. Most of the THz power is propagating in the cladding with low $\mathrm{THz}$ absorption, thereby reducing losses. In case of $\mathrm{LN}$ core, it was shown that a smaller pulse front tilt angle is required for velocity matching than in bulk $\mathrm{LN}$, thereby increasing the interaction length and hence the conversion efficiency. Practical considerations and expected performance were presented for the design of an ARWG THz source for a broad range of laser pulse energies and repetition rates.

The ARWG structure will expectedly enable highly efficient $\mathrm{THz}$ pulse generation in highly nonlinear materials having large absorption coefficient by using moderate pump energy delivered, for example, by compact fiber laser sources. The predicted more than one order of magnitude increase in the conversion efficiency opens up the possibility to build highly efficient and compact $\mathrm{THz}$ sources with extremely high average output power. Such sources can find many applications in (nonlinear) THz spectroscopy, imaging, and security.

Financial support from Hungarian Scientific Research Fund (OTKA) Grant No. 113083 is acknowledged. J.A.F. acknowledges support from János Bolyai Research Scholarship (Hungarian Academy of Sciences). The present scientific contribution is dedicated to the 650th anniversary of the foundation of University of Pécs, Hungary.

${ }^{1}$ S. B. Bodrov, A. N. Stepanov, M. I. Bakunov, B. V. Shishkin, I. E. Ilyakov, and R. A. Akhmedzhanov, Opt. Express 17, 1871 (2009).

${ }^{2}$ M. I. Bakunov, B. Bodrov, and M. Hangyo, J. Appl. Phys. 104, 093105 (2008).

${ }^{3}$ S. B. Bodrov, I. E. Ilyakov, B. V. Shishkin, and A. N. Stepanov, Appl. Phys. Lett. 100, 201114 (2012).

${ }^{4}$ M. I. Bakunov, E. A. Mashkovich, M. V. Tsarev, and S. D. Gorelov, Appl. Phys. Lett. 101, 151102 (2012).

${ }^{5}$ K. Suizu, K. Koketsu, T. Shibuya, T. Tsutsui, T. Akiba, and K. Kawase, Opt. Express 17, 6676 (2009).

${ }^{6}$ W. Shi and Y. Ding, Appl. Phys. Lett. 82, 4435 (2003).

${ }^{7}$ A. G. Stepanov, J. Hebling, and J. Kuhl, Appl. Phys. B 81, 23 (2005).

${ }^{8}$ K. L. Vodopyanov and Y. Avetisyan, Opt. Lett. 33, 2314 (2008).

${ }^{9}$ R. Zhichao, V. Georgios, K. L. Vodopyanov, M. M. Fejer, and S. Fan, Opt. Express 17, 13502 (2009).

${ }^{10}$ J. Hebling, K. L. Yeh, M. C. Hoffmann, B. Bartal, and K. A. Nelson, J. Opt. Soc. Am. B 25, 6 (2008).

${ }^{11}$ L. Pálfalvi, J. Hebling, J. Kuhl, Á. Péter, and K. Polgár, J. Appl. Phys. 97, 123505 (2005).

${ }^{12}$ J. Hebling, G. Almási, I. Kozma, and J. Kuhl, Opt. Express 10, 1161 (2002).

${ }^{13}$ J. A. Fülöp, L. Pálfalvi, G. Almási, and J. Hebling, Opt. Express 18, 12311 (2010).

${ }^{14}$ O. Gayer, Z. Sacks, E. Galun, and A. Arie, Appl. Phys. B 91, 343 (2008).

${ }^{15}$ See http://www.tydexoptics.com/products/thz_optics/thz_materials/ for refractive indices and THz absorption coefficient of PMP.

${ }^{16}$ D. R. Grischkowsky, Opt. Photonics News 3, 21 (1992).

${ }^{17}$ Y. S. Jin, G. J. Kim, and S. G. Jeon, J. Korean Phys. Soc. 49, 513 (2006).

${ }^{18}$ B. E. A. Salech and M. C. Teich, Fundamentals of Photonics (John Wiley \& Sons, Inc., 1991).

${ }^{19}$ J. A. Fülöp, L. Pálfalvi, M. C. Hoffmann, and J. Hebling, Opt. Express 19, 15090 (2011).

${ }^{20}$ W. Schneider, A. Ryabov, Cs. Lombosi, T. Metzger, Zs. Major, J. A. Fülöp, and P. Baum, Opt. Lett. 39, 6604 (2014).

${ }^{21}$ K. Ravi, W. R. Huang, S. Carbajo, W. Wu, and F. Kärtner, Opt. Express 22, 20239 (2014). 